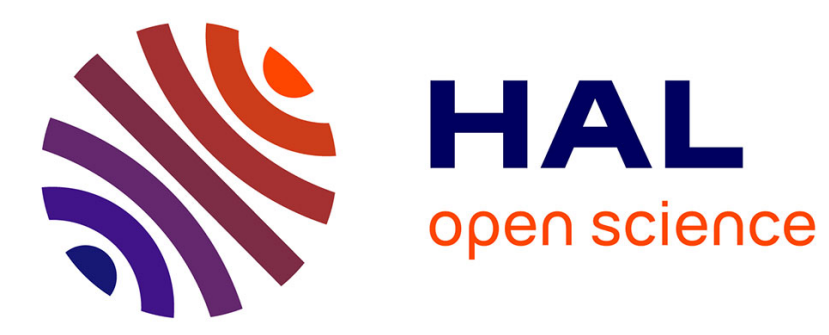

\title{
Remote Stabilization via Time-Varying Communication Network Delays: Application to TCP networks
}

Emmanuel Witrant, Carlos Canudas de Wit, Didier Georges, Mazen Alamir

\section{To cite this version:}

Emmanuel Witrant, Carlos Canudas de Wit, Didier Georges, Mazen Alamir. Remote Stabilization via Time-Varying Communication Network Delays: Application to TCP networks. IEEE Conference on Control Applications, Sep 2004, Taiwan. hal-00196872

\section{HAL Id: hal-00196872 \\ https://hal.science/hal-00196872}

Submitted on 13 Dec 2007

HAL is a multi-disciplinary open access archive for the deposit and dissemination of scientific research documents, whether they are published or not. The documents may come from teaching and research institutions in France or abroad, or from public or private research centers.
L'archive ouverte pluridisciplinaire HAL, est destinée au dépôt et à la diffusion de documents scientifiques de niveau recherche, publiés ou non, émanant des établissements d'enseignement et de recherche français ou étrangers, des laboratoires publics ou privés. 


\title{
Remote Stabilization via Time-Varying Communication Network Delays: Application to TCP networks
}

\author{
E. Witrant, C. Canudas-de-Wit, D. Georges, and M. Alamir \\ Laboratoire d'Automatique de Grenoble, UMR CNRS 5528 \\ ENSIEG-INPG, B.P. 46, 38 402, Saint Martin d’Heres, FRANCE
}

\begin{abstract}
In this paper we investigate the problem of remote stabilization via communication networks. This problem arises when the control law is remotely implemented. The exchange of data between the controller and the system is done via a data communication network described by a deterministic model. This leads to the problem of stabilizing an open-loop unstable system with time-varying delay. Assuming a known general model for the time-delay dynamics, we develop a time-varying horizon predictor, which is used as a basis to build a stabilizing control law that includes explicitly the delay dynamics. This control law is then applied to the case of TCP networks. Experimental results are presented.
\end{abstract}

Index Terms-Networked control systems, stabilization with time-varying delays, TCP networks.

\section{INTRODUCTION}

The networked control systems constitute a new class of control systems including specific problems such as delays, loss of information and data process. The problem studied in this paper concerns the remote stabilization of unstable open-loop systems. The control setup is shown in Figure (1). The sensor, actuator and system are assumed to be remotely commissioned by a controller that interchange measurements and control signals through a communication network. This network is used by multiple systems that emit on the same communication channel and a router is introduced to manage and distribute the information. A Transfer Protocol (TP) is implemented to allow users to send and receive data over the network. Examples of such protocols are:

- User Data Protocol (UDP)

- Transfer Control Protocol (TCP)

- Network Control Protocol (NCP)

- Sequenced Packet Exchange (SPX)

The first two are built on Internet Protocol (IP) networks while NCP/SPX work on Internetwork Packet Exchange (IPX) networks. Note that TCP and SPX are secure protocol protocols, meaning that the lost packets are re-emitted. The impact of such network is to introduce a time-varying delay in the data transmission between the system and the controller, due to the multiple users interaction.

The time-varying delay makes the problem more difficult since the time-translation is not reversible. Most of the existing control methods (like the Lyapunov-Krasovskii approaches) either assume a constant time-delay, or a known upper bound on it together with the assumption that the time-derivative of

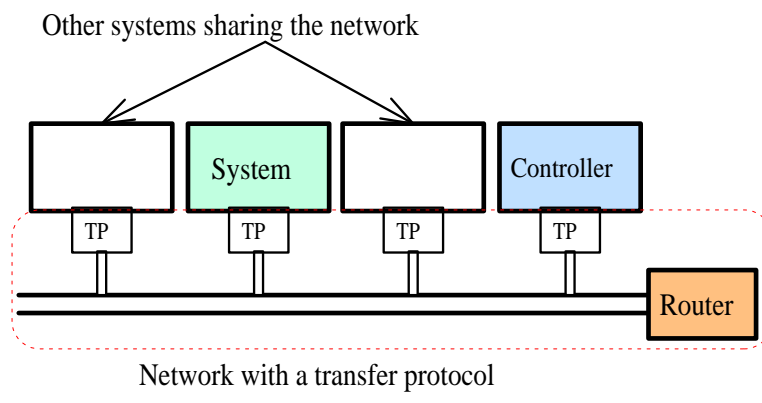

Fig. 1. Closed-loop network controlled system.

the delay is bounded by 1 (see for example [1] or [2]). The case of time-varying or state-dependent delays can be treated along the solutions presented in [3], and [4] as long as the system is open-loop stable. These solutions do not allow for a direct use of the time-delay dynamics in the design of the control law and naturally yield to conservative results.

The case of open-loop unstable systems with time-varying delays has not been fully studied yet, but it may be of interest in some tele-operation problems. An example is the airplane drones, that are remotely controlled and open-loop unstable, to allow for a faster response. Another example is a complex system such as a supply chain, where all the subsystems and controllers are connected together through a local area network.

The stabilization of an open-loop unstable system with timevarying delays is studied in [5] and [6], where the system is stabilized with a state predictor and a precise condition on the time-varying predictor's horizon is established. This predictor is included in a $H^{\infty}$ control scheme in [7] and results in a LMI formulation. In [8], the state predictor is used to stabilize a system under two channels time-varying delays and to design an observer-based control; the results of this work can be applied directly with the present control scheme if the delayed output is to be used to establish the control law. In this paper, we use this control structure, based on the ideas of "poleplacement", and show precisely how the available information on the communication network dynamics is involved in the computation of the predictor's horizon. We show that, under certain weak conditions, we are able to set the eigenvalues of a time-varying shifted system, or equivalently we transform 
the time-invariant delayed unstable open-loop system, into a stable time-varying linear system.

\section{Problem Statement}

The aim of this paper is then to explore how the transmission protocol dynamics can be explicitly used in the design of the control feedback. These dynamics can be described by the general class of systems that write as

$$
\begin{aligned}
& \dot{z}(t)=f\left(z(t), u_{d}(t)\right), \quad z(0)=z_{0} \\
& \tau(t)=h\left(z(t), u_{d}(t)\right)
\end{aligned}
$$

where

- $z(t)$ is the internal state of the network (with initial state $z_{0}$ ), that describes the time evolution of the emitters window size $W_{i}(t)$ (for $i=1 \ldots N$ sources connected to the network) and the router's queue length $q(t)$, for example. In that case, the state writes as $z(t)=$ $\left[\begin{array}{llll}W_{1}(t) & \ldots & W_{N}(t) & q(t)\end{array}\right]^{T}$

- $u_{d}(t)$ is the exogenous input to the system, which is the number of users and the router's capacity $C_{r}$, if both are time-varying. We then have $u_{d}(t)=\left\{N, C_{r}\right\}$,

- $f\left(z(t), u_{d}(t)\right)$ describes the internal dynamics of the network, set by the TP on the window sizes, and by the queue management scheme on the queue length,

- $h\left(z(t), u_{d}(t)\right)$ gives the resulting delay $\tau(t)$ from the whole model. This delay is the one that occurs from the controller to the source.

Note that (1)-(2) describe an autonomous system with an exogenous input $u_{d}(t)$. This input is assumed to be known over a certain range of time ahead of the present time (equal to the maximum delay expected $\tau_{\max }$ ). This would be the case if the subsystems of a supply chain act in a predetermined order or if the transfer protocol is set to declare to the network that its source will emit and wait during $\tau_{\max }$ before starting the emission.

An example of such dynamics is the TCP model described by [9], where a fluid flow model was developed using Poisson counter driven differential equations, with a proportional Active Queue Management (AQM) policy set on the router's site. The AQM is introduced with a packet discard function $p(x)$ and acts as a feedback from the router on the emitter's window size; the proportional scheme is shown to be stable in [10]. The network equations then write as

$$
\begin{aligned}
\frac{d W_{i}(t)}{d t} & =\frac{1}{R_{i}(t)}-\frac{W_{i}(t)}{2} \frac{W_{i}\left(t-R_{i}(t)\right)}{R_{i}\left(t-R_{i}(t)\right)} p_{i}(t), \\
\frac{d q(t)}{d t} & =-C_{r}+\sum_{i=1}^{N} \frac{W_{i}(t)}{R_{i}(t)}, \quad q\left(t_{0}\right)=q_{0} \\
\tau_{i} & =\frac{1}{2}\left[\frac{q(t)}{C_{r}}+T_{p i}\right]
\end{aligned}
$$

where $R_{i}(t) \doteq \frac{q(t)}{C_{r}}+T_{p i}$ is the round trip time, $p_{i}(t)=$ $K_{p} q\left(t-R_{i}(t)\right), T_{p i}^{r}$ is the constant propagation delay.

The remotely controlled system has the form:

$$
\begin{aligned}
\dot{x}(t) & =A x(t)+B u(t-\tau(t)) \\
y(t) & =C x(t)
\end{aligned}
$$

where $x \in R^{n}$ is the internal state, $u \in R$ is the control input, $y \in R^{m}$ is the system output, and $A, B, C$ are matrices of appropriate dimensions. The pairs $(A, B)$ and $(A, C)$ are assumed to be controllable and observable, respectively, but no assumption is made on the stability of $A$. We assume that all solutions of model (1)-(2), lead to the following property

$$
\begin{array}{r}
0 \leq \tau(t) \leq \tau_{\max }, \quad \forall t \geq 0 \\
\dot{\tau}(t)<1, \quad \text { for almost all } t \geq 0
\end{array}
$$

where $\tau_{\max } \geq 0$ is an upper bound on the time-delay. Note that condition (8) is a technical one, first introduced in [5] and necessary for the stability analysis.

\section{CONTROL DESIGN}

We consider the simple problem of state feedback stabilization, where we assume that the full state is measurable, i.e. $y(t)=x(t)$. Defining a new input $v(t)$ as

$$
v(t)=u(t-\tau(t)),
$$

assuming the existence of a bounded time-depending function $\infty>\delta(t) \geq 0$ (to be defined later), and replacing the current time $t$ by the shifted time coordinate $t+\delta(t)$ in (5), we have

$$
\dot{x}(t+\delta(t))=A x(t+\delta(t))+B v(t+\delta(t))
$$

It is worth to note that for time-varying delays, the time translation is not reversible, i.e.

$$
v(t+\delta(t))=u(t-\tau(t+\delta(t))+\delta(t))
$$

as it is the case when the delay is constant, and $\delta=\tau$.

Assume that it is possible to set

$$
v(t+\delta(t))=-K x(t+\delta(t))
$$

Then the resulting closed-loop equation is

$$
\dot{x}(t+\delta(t))=(A-B K) x(t+\delta(t))=A_{c l} x(t+\delta(t))
$$

where $A_{c l}$ is the closed loop state matrix, that can be made negative definite, by the controllability hypothesis on the $(A, B)$ pair. Nevertheless, stability of this system does not follow straightforwardly from the stability of the matrix $A_{c l}$, as it is the case for systems with constant time-delay. We will come to this point, after presenting the procedure to predict $v(t+\delta(t))$, and the resulting constructive definition for $\delta(t)$. To sum up, two conditions are to be satisfied for the above scheme to be implementable

1) The possibility to predict $x(t+\delta(t))$

2) The possibility to assign $v(t+\delta(t))$. From (9), this is possible provided that $t-\tau(t+\delta(t))+\delta(t)=t$. This leads to a necessary condition on $\delta(t)$ that will be used later.

The prediction of $x(t+\delta(t))$ is then the key point for the construction of (10), and for (11) to hold. Introducing (10), in

$$
\begin{aligned}
x(t+\delta(t)) & =e^{A \delta(t)} x(t) \\
& +e^{A(t+\delta(t))} \int_{t}^{t+\delta(t)} e^{-A \theta} B u(\theta-\tau(\theta)) d \theta
\end{aligned}
$$


gives

$$
\begin{aligned}
v(t+\delta(t)) & =-K e^{A \delta(t)} x(t) \\
& -K e^{A(t+\delta(t))} \int_{t}^{t+\delta(t)} e^{-A \theta} B u(\theta-\tau(\theta)) d \theta
\end{aligned}
$$

Using (9) in the above equation yields

$$
\begin{aligned}
& u(t-\tau(t+\delta(t))+\delta(t))=-K e^{A \delta(t)} x(t) \\
& -K e^{A(t+\delta(t))} \int_{t}^{t+\delta(t)} e^{-A \theta} B u(\theta-\tau(\theta)) d \theta
\end{aligned}
$$

The argument of the integral involves information from $u(t-$ $\tau(t))$ to $u(t+\delta(t)-\tau(t+\delta(t)))$. Thus, causality in the computation of the control law holds if the following condition holds

$$
\forall \theta \in[t, t+\delta(t)], \quad \theta-\tau(\theta) \leq t
$$

That is the reason why $\delta(t)$ is defined as follows

$$
\delta(t) \doteq \max \{\delta \geq 0 \quad \mid \forall \theta \in[t, t+\delta], \quad \theta-\tau(\theta) \leq t\}
$$

The existence of solution to (13) is shown by the following proposition

Proposition 3.1: The optimization problem (13) admits a solution $\delta(t)$ for which

$$
\delta(t)-\tau(t+\delta(t))=0
$$

Proof Let $t$ be some given instant. The admissible set for the optimization problem (13) is non empty since the value $\delta=0$ is a candidate solution that meets the constraint $t-\tau(t) \leq t$ [This is because $\tau(t) \geq 0$ by assumption (7)]. Furthermore, the admissible set is bounded since an upper bound is given by $\delta=\sup _{\sigma \geq 0} \tau(\sigma)$. Finally, by classical continuity arguments, the admissible set is clearly closed. Therefore, the optimization problem (13) is that of finding the maximal value of a continuous function (the identity map) over a compact set. The fact that the solution $\delta(t)$ satisfies (14) is a direct consequence of its maximal property. $\diamond \diamond \diamond$

Based on the above proposition, it is clear that $\delta(t)$ defined by (13) clearly enables (12) to be written in the following computable form

$$
\begin{aligned}
u(t)= & -K e^{A \delta(t)}[x(t) \\
& \left.+e^{A t} \int_{t}^{t+\delta(t)} e^{-A \theta} B u(\theta-\tau(\theta)) d \theta\right]
\end{aligned}
$$

since it only uses past information of $u(t)$ in the time interval $[t-\tau(t), t]$.

Remark. During the implementation of control law (15), it is necessary to continuously solve equation (14) for $\delta(t)$ at all times and to keep a history of the past control inputs during a time-interval $[t-\tau(t), t]$. This is detailed in a subsequent section

\section{A. Stability analysis}

Lemma 3.1: Consider the following system

$$
\frac{d x}{d t}(t+\delta(t))=A_{c l} x(t+\delta(t))
$$

for $t \geq 0$ and $\delta(0)=\delta_{0}$. Then, if the following conditions holds:

i) all the real part of the eigenvalues of $A_{c l}$ are in the open left hand side of the complex plane,

ii) $\infty>\delta_{M} \geq \delta(t) \geq 0$,

iii) $\infty>\rho>\dot{\delta}(t)>-1$.

then,

$$
\lim _{t \rightarrow \infty}\|x(t+\delta(t))\|=0, \quad \forall t \geq \delta_{0}
$$

and for all bounded values of $x\left(\delta_{0}\right)$.

Proof. Introduce $\zeta(t)=t+\delta(t)$, then (16) writes as

$$
\begin{aligned}
\frac{d x(\zeta)}{d \zeta} \frac{d \zeta}{d t} & =A_{c l} x(\zeta) \\
\frac{d x(\zeta)}{d \zeta} & =\gamma(t) A_{c l} x(\zeta), \quad \gamma(t)=\frac{1}{1+\frac{d \delta(t)}{d t}}
\end{aligned}
$$

Note that this equation describes a linear time-variant system in the shifted time-coordinates $\zeta(t)$.

Introducing the Lyapunov function

$$
V(\zeta)=x(\zeta)^{T} \operatorname{Px}(\zeta)
$$

we get together with the assumption $(i)$, that there exist a positive definite matrix $Q$ such that

$$
\begin{aligned}
\frac{d V(\zeta)}{d \zeta} & =x(\zeta)^{T}\left(P A_{c l}+A_{c l}^{T} P\right) x(\zeta) \gamma(t) \\
& =-x(\zeta)^{T} Q x(\zeta) \gamma(t) \\
& \leq-\lambda_{m}(Q)\|x(\zeta)\|^{2} \gamma(t)<0
\end{aligned}
$$

where the last inequality comes from the assumption (iii). Using the bounds $\lambda_{M}(P)\|x(\zeta)\|^{2} \geq V(\zeta) \geq \lambda_{m}(P)\|x(\zeta)\|^{2}$, and integrating this inequality from $\zeta(0)=\delta_{0}$ to $\zeta(t)$, we get

$$
V(\zeta) \leq V\left(\delta_{0}\right) e^{-\Psi\left(\delta_{0}, \zeta\right)}
$$

where

$$
\Psi\left(\delta_{0}, \zeta\right)=\frac{\lambda_{m}(Q)}{\lambda_{M}(P)} \int_{\delta_{0}}^{\zeta} \gamma(\theta) d \theta>0
$$

from which we obtain the relation

$$
\|x(\zeta)\|^{2} \leq \frac{\lambda_{m}(P)}{\lambda_{M}(P)}\left\|x\left(\delta_{0}\right)\right\|^{2} e^{-\Psi\left(\delta_{0}, \zeta\right)},
$$

It remain to be shown that $\Psi\left(\delta_{0}, \zeta\right) \rightarrow \infty$ as $t \rightarrow \infty$. For this, we use the last assumption to show that

$$
\Psi\left(\delta_{0}, \zeta\right) \geq \frac{\lambda_{m}(Q)}{\lambda_{M}(P)} \int_{\delta_{0}}^{\zeta} \frac{1}{1+\rho} d \theta=\frac{\lambda_{m}(Q)}{\lambda_{M}(P)} \frac{1}{1+\rho}\left(\zeta-\delta_{0}\right)
$$

From the boundedness of $\delta(t)$ and $\rho$, we have that $t \rightarrow \infty$ implies that $\zeta \rightarrow \infty$ as well, then from the previous relation, we have

$$
\lim _{t \rightarrow \infty} \Psi\left(\delta_{0}, \zeta\right) \geq \lim _{t \rightarrow \infty} \frac{\lambda_{m}(Q)}{\lambda_{M}(P)} \frac{1}{1+\rho}\left(\zeta-\delta_{0}\right)=+\infty
$$

then $\lim _{t \rightarrow \infty} \Psi\left(\delta_{0}, \zeta\right)=+\infty$, which applied to the relation (17), concludes the proof.

$\diamond \diamond \diamond$

Corollary 3.1: the control law (15) applied to the system (5)-(6), has a bounded solution and exponentially converges to zero, for all $t \geq 0$. 
Proof. System (5)-(6) is a linear one, and its states cannot diverge in finite time. Therefore for any bounded $\delta_{0}$, the state at that time $x\left(\delta_{0}\right)$ will be bounded. From the previous lemma, the state will then exponentially converge to zero.

$\diamond \diamond \diamond$

\section{B. Computation of $\delta(t)$ and use of the time-delay model}

In this subsection we discuss several aspects related to the computation of the prediction time-varying horizon, and provide some special cases where this computation can be donne explicitly.

The computation of the control law implies to continuously solve, for $\delta(t)$, the implicit equation (14) for at all $t \geq 0$. The existence of a solution of this equation is not trivial and implies that $\tau($.$) satisfies some particular conditions.$

This a problem of the type $\delta=g(\delta, t)$, where $g(\delta, t)=$ $\tau(t+\delta)$. The existence of a solution to this problem is first established by considering the special case where the time is frozen (i.e. $g(\delta, t)=g(\delta)$ ), and then extended to the general case by noticing that this remains true for all times.

Let $\mathcal{C}\left(R^{n+},[a, b]\right)$ define a class of continuous function mapping $R^{n+} \mapsto[a, b]$. From the definition of the admissible $\tau(\cdot)$ considered in this paper; continuous and bounded functions, and the fact that $t \in R^{+}$, and $0 \leq \tau(t) \leq \tau_{\max }$, then we have that $\tau(t) \in \mathcal{C}\left(R^{+},\left[0, \tau_{\text {max }}\right]\right)$.

Then, considering also the fact that $g(\delta, t)$ maps $R^{2+}$ into $R^{+}$, the following property is established

$$
g(\delta, t) \in \mathcal{C}\left(R^{2+},\left[0, \tau_{\max }\right]\right) \forall \delta, t \in R^{+}
$$

Finally from the fact that $\delta=g(\delta, t)$, we have that

$$
\delta \in\left[0, \tau_{\max }\right]
$$

a) Existence of a solution: The fixed point theorem states that if $g$ is a continuous function $g(\sigma) \in[a, b]$ for all $\sigma \in[a, b]$, then $g$ has a fixed point in $[a, b]$.

This theorem is applied to the problem of the existence of $\delta$ by first considering $g\left(\delta, t_{1}\right)$, where $t_{1}>0$ is a given time instant. In that case the fixed point theorem, along with the properties on $g$ and $\delta$ given by (18)-(19), directly ensures that there exists a fixed point $\delta \in\left[0, \tau_{\max }\right]$. This is then extended to the general case by noticing that this remains true for all $t_{1} \in R^{+}$. Therefore, we can ensure that there exists a $\delta(t) \in$ $\left[0, \tau_{\max }\right]$ such that (14) is satisfied. This means the following

Result 3.1: If the assumption boundedness and continuity $C^{1}$ on the time-variation of the delay are respected, then is always a solution for $\delta$ satisfying equation (14).

b) Solution search: It is clear that a complete model of the time delay is needed to compute $\delta(t)$, at least over a time interval $[0, t+\delta(t)]$. This solution can be obtained by different methods, depending on the structure of the delay's model and the available information:

- A particular case where an explicit solution for $\delta$ do exist, is given by the following exemple. Assume that the delay differential equation is given by

$$
\begin{aligned}
\dot{z}(t) & =a_{t} z(t)+b_{t}, \quad \text { with } \quad z(0)=z_{0} \\
\tau(t) & =c_{t} z(t)
\end{aligned}
$$

with $a_{t}, b_{t}, c_{t}$ and $z_{0}$ being scalar constants. In that case, the explicit solution of (14) can be established and solved analytically using Lambert's W function [11]. This results in the following solution

$$
\delta(t)=-\left[W\left(-a_{t} c_{t} z_{0} e^{a_{t} t-c_{t} b_{t}}\right)+c_{t} b_{t}\right] / a_{t}
$$

- For the multi-variable case with time varying inputs; i.e. if

$$
h\left(z(t), u_{d}(t)\right)=z(t)
$$

then

$$
\delta(t)=z_{0}+\int_{0}^{t+\delta} f\left(z(\theta), u_{d}(\theta)\right) d \theta
$$

can be found by explicit numerical search.

- The dichotomy method can be applied on (14) using the numerical values of the time delay: the validity of this method follows from the fixed point theorem but is time consuming since the dichotomy is used at each sampling time,

- The delay dynamics can be used directly by first defining the function $S(t)=\delta(t)-\tau(t+\delta(t))$ and then finding $\dot{\delta}(t)$ such that $\delta(t)$ reaches asymptotically the manifold $S(t)=$ 0 . In order to prevent for the numerical instabilities, the dynamics of $S(t)$ is defined as

$$
\dot{S}(t)+\lambda S(t)=0
$$

where $\lambda$ is a positive constant. (20) is satisfied if $\dot{\tau} \neq 1$ and $\delta(t)$ has the following dynamics

$$
\dot{\delta}(t)=-\frac{\lambda}{1-d \tau(\zeta) / d \zeta} \delta+\frac{d \tau(\zeta) / d \zeta+\lambda \tau(\zeta)}{1-d \tau(\zeta) / d \zeta}
$$

where $\zeta=t+\delta$. Note that the initial condition $\delta(0)$ doesn't need to be known and can be chosen arbitrarily in $\left[0, \tau_{\max }\right]$ since $\lambda$ ensures the asymptotic convergence of the solution. To illustrate the computation of $\dot{\delta}$, consider the exemple presented above, where $h()=.z(t)$ : in that case $d \tau(\zeta) / d \zeta=f\left(z(t+\delta), u_{d}(t+\delta)\right)$.

The main contribution of the paper is now summarized in the following theorem.

Theorem 3.1: Consider the system

$$
\dot{x}(t)=A x(t)+B u(t-\tau(t))
$$

$(A, B)$ controllable pair. Assume that the delay dynamics (1)(2) is such that the following holds for $\tau(t)$

A1) $\tau(t) \in \mathcal{C}\left(R^{+},\left[0, \tau_{\text {max }}\right]\right) \quad \forall t$,

A2) $\dot{\tau}(t)<1 \quad \forall t \geq t_{0}$.

Then the feedback control law

$$
\begin{aligned}
u(t) & =-K e^{A \delta(t)}\left[x(t)+e^{A t} \int_{t}^{t+\delta(t)} e^{-A \theta} B u(\theta-\tau(\theta)) d \theta\right] \\
\dot{\delta}(t) & =-\Phi_{1}\left(\frac{d \tau(\zeta)}{d \zeta}\right) \delta+\Phi_{2}\left(\frac{d \tau(\zeta)}{d \zeta}, \tau(\zeta)\right) \\
\frac{d \tau}{d \zeta}(\zeta) & =\frac{d h}{d \zeta}\left(z(\zeta), u_{d}(\zeta)\right) \\
\frac{d z}{d \zeta}(\zeta) & =f\left(z(\zeta), u_{d}(\zeta)\right), \quad z(0)=z_{0}
\end{aligned}
$$


with $\zeta=1+\delta, \lambda$ a positive constant, $\delta(0)=\delta_{0}$, and

$$
\begin{aligned}
\Phi_{1}(d \tau(\zeta) / d \zeta) & =\frac{\lambda}{1-d \tau(\zeta) / d \zeta} \\
\Phi_{2}(d \tau(\zeta) / d \zeta, \tau(\zeta)) & =\frac{d \tau(\zeta) / d \zeta+\lambda \tau(\zeta)}{1-d \tau(\zeta) / d \zeta}
\end{aligned}
$$

ensures that the closed-loop system is bounded, and that the state $x(t)$ converges exponentially to zero.

Proof. Note that thanks to assumption $A 1$, proposition 3.1 holds. Then hypothesis (iii), along with the time derivative of (14) and the fact that the properties on $\dot{\tau}(t)$ can be extended to $d \tau(\zeta) / d \zeta$ for all $\zeta \in R^{+}$, implies that

$$
-1<\frac{\dot{\tau}(t)}{1-\dot{\tau}(t)}<\rho
$$

when $\dot{\tau}(t) \neq 1$. It can be easily verified that the lower bound is always true and doesn't imply any constraint on $\dot{\tau}$, while the upper bound condition is given by hypothesis $(A 2)$ since $\rho$ can be chosen arbitrarily large. Therefore, the proof follows using the previous lemma and corollary, and by noticing that (A2) implies (iii). The asymptotic convergence of $\delta(t)$ is obtained from the positiveness of $\lambda$ and $(A 2)$ since it ensures that $\Phi_{1}(\cdot)>0$ for all $t$.

\section{Application to the TCP network}

We now detail how the TCP model described by (3)-(4) is used in the computation of $\delta(t)$ to set the control law established in Theorem 3.1. We consider the special case where the router's capacity is constant. From the definition of the $R_{i}(t)$, we have that

$$
\tau(\zeta)=\frac{1}{2}\left[\frac{q(\zeta)}{C_{r}}+T_{p c s}\right]
$$

Deriving the previous equation along with (4), it follows that

$$
\frac{d \tau}{d \zeta}(\zeta)=\frac{1}{2 C_{r}}\left[\sum_{i=1}^{N(\zeta)} \frac{W_{i}(\zeta)}{R_{i}(\zeta)}-C_{r}\right]
$$

where $R_{i}(\zeta)$ is obtained from $q(\zeta), N(\zeta)$ is assumed to be known. Both $W_{i}(\zeta)$ and $q(\zeta)$ are obtained from the dynamics (3)-(4): this is done by continuously computing the solutions of (3)-(4) up to the time $t+\tau_{\max }$. (23)-(24) can now be substituted in (21)-(22) to obtain the dynamics $\dot{\delta}(t)$.

\section{EXPERIMENTAL RESULTS}

We describe in this section how a "T-shape" inverted pendulum, depicted on fig. 2, is controlled through a TCP network. The detailed model description of this system can be found in [12] and is briefly recalled here. The inverted pendulum is composed of an horizontal actuated rode that can slide on the top of a vertical rod; the bottom of the vertical rod rotates freely around a point fixed on the supporting structure. The angle between the vertical upward position and the vertical rod is $\theta(t)$, measured positive counterclockwise, and the displacement of the horizontal rod from the central position is $z(t)$, positive to the left. This system is actuated

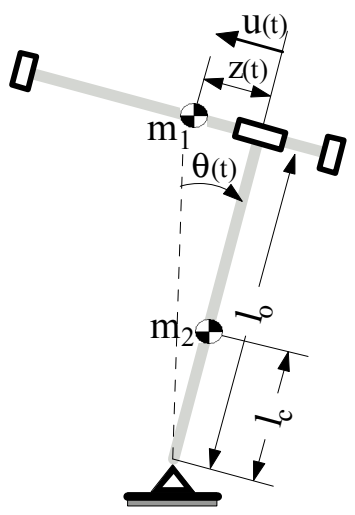

Fig. 2. The inverted pendulum considered.

by applying a linear force $u(t)=F(t)$ to the horizontal rod using a d.c. motor placed at the bottom of the vertical rod and coupled to the horizontal one through transmission mechanics; thanks to two encoders, $z(t)$ and $\theta(t)$ are measured. The position of the vertical rod center of gravity can be moved upward or downward by setting the height of a mass placed at the bottom of the rod.

The nonlinear model (without friction) is described by

$$
\begin{aligned}
& {\left[\begin{array}{cc}
m_{1} & m_{1} l_{0} \\
m_{1} l_{0} & \bar{J}+m_{1} z^{2}
\end{array}\right]\left[\begin{array}{c}
\ddot{z} \\
\ddot{\theta}
\end{array}\right]+\left[\begin{array}{cc}
0 & -m_{1} z \dot{\theta} \\
2 m_{1} z \dot{\theta} & 0
\end{array}\right]\left[\begin{array}{c}
\dot{z} \\
\dot{\theta}
\end{array}\right]} \\
& +\left[\begin{array}{c}
1 \\
-\left(m_{1} l_{0}+m_{2} l_{c}\right) \sin \theta
\end{array}\right] u,
\end{aligned}
$$

where the time dependance of the state variables is implicit, and the parameters meaning and values are listed in the following table.

\begin{tabular}{|c|l|l|}
\hline Parameter name & Value & Meaning \\
\hline$m_{1}$ & $0.213 \mathrm{~kg}$ & Mass of the horizontal rod. \\
$m_{2}$ & $1.785 \mathrm{~kg}$ & Mass of the vertical rod. \\
$l_{0}$ & $0.33 \mathrm{~m}$ & Length of the vertical rod. \\
$l_{c}$ & $-0.029 \mathrm{~m}$ & Vertical rod c.g. position. \\
$g$ & $9.807 \frac{\mathrm{m}}{\mathrm{s}^{2}}$ & Gravity acceleration. \\
$\bar{J}$ & $0.055 \mathrm{Nm} \mathrm{m}^{2}$ & Nominal momentum of inertia. \\
\hline
\end{tabular}

Defining the state vector as $x \doteq[z, \dot{z}, \theta, \dot{\theta}]^{\top}$, linearization around the equilibrium point $\bar{x}=0$ under $u(t)=0$, gives a state space representation in the standard form $\dot{x}(t)=A x(t)+$ $B u(t), y(t)=x(t)$, where

$$
A=\left[\begin{array}{cccc}
0 & 1 & 0 & 0 \\
-21.54 & 0 & 14.96 & 0 \\
0 & 0 & 0 & 1 \\
65.28 & 0 & -15.59 & 0
\end{array}\right], \quad B=\left[\begin{array}{c}
0 \\
8.10 \\
0 \\
-10.31
\end{array}\right]
$$

The eigenvalues of $A$ are $\lambda_{1,2}= \pm 7.07 \mathrm{\jmath}, \lambda_{3}=3.58$ and $\lambda_{4}=-3.58$; the two pure imaginary eigenvalues depend on the vertical rod dynamics without friction, while the positive real one depends on the sliding rod dynamics. The linearized model of the pendulum is used to build the control law and the controller gain $K$ is chosen so that the closed-loop poles of the time-shifted system are set to $[-8+0.5 i ;-8-0.5 i ;-16 ;-32]$.

The network consists of one router and two TCP flows (the one used by the system and the controller, and a disturbing one, acting between $t=10 \mathrm{~s}$ and $t=25 \mathrm{~s}$ ). Its parameters 

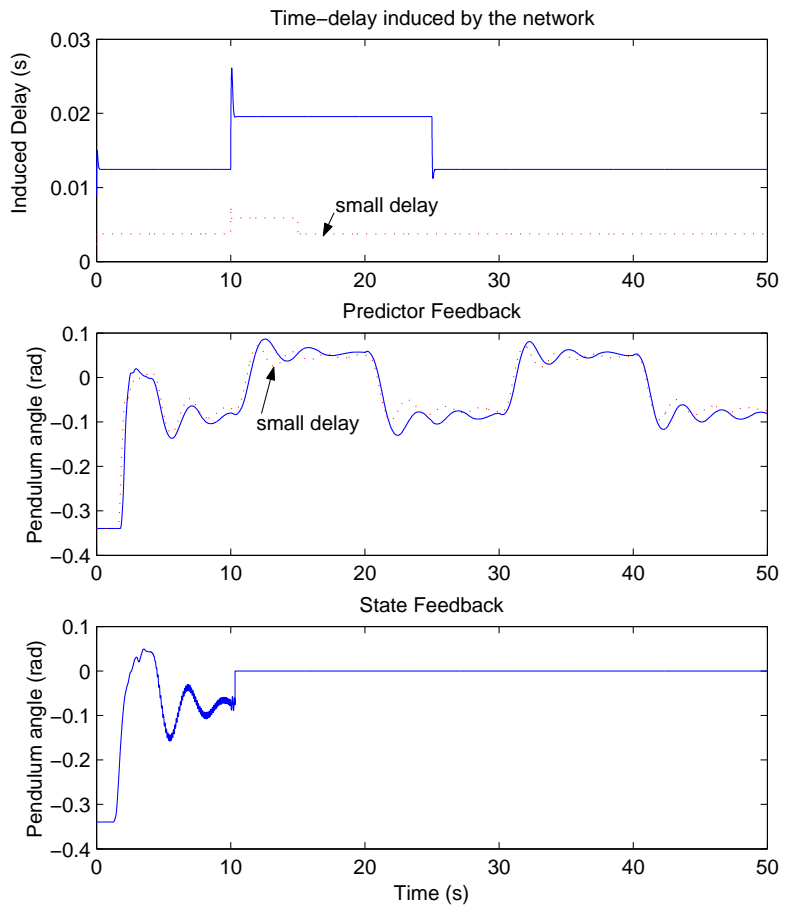

Fig. 3. Inverted pendulum's response to a filtered square reference.

are such that the time-delay is obtained from the following dynamics

$$
\begin{aligned}
\frac{d W_{1}(t)}{d t} & =\frac{1}{R_{1}(t)}-\frac{W_{1}(t)}{2} \frac{W_{1}\left(t-R_{1}(t)\right)}{R_{1}\left(t-R_{1}(t)\right)} p_{1}(t), \\
\frac{d W_{2}(t)}{d t} & =\frac{1}{R_{2}(t)}-\frac{W_{2}(t)}{2} \frac{W_{2}\left(t-R_{2}(t)\right)}{R_{2}\left(t-R_{2}(t)\right)} p_{2}(t), \\
\frac{d q(t)}{d t} & =-300+\sum_{i=1}^{2} \frac{W_{i}(t)}{R_{i}(t)}, \quad q(0)=5 \\
\tau(t) & =R_{1}(t) / 2
\end{aligned}
$$

with $R_{1}(t) \doteq \frac{q(t)}{300}+0.001, R_{2}(t) \doteq \frac{q(t)}{300}+0.0015, p_{1,2}(t)=$ $0.005 q\left(t-R_{1,2}(t)\right)$, and $W_{1}(0)=W_{2}(10)=10$ packets. The computation of $\delta(t)$ and the control law is then straightforward from (23)-(24) and Theorem 3.1.

The time-delay induced by the proposed network model and the resulting $\delta(t)$ are pre-computed, while the control law is implemented in a discrete form (the integral part is approximated using the backward rectangular rule). The sampling time of the sensors, actuator and control is set to $1 \mathrm{~ms}$, and the inverted pendulum's response to a filtered square reference is studied. The induced time-delay, the closed-loop response with the proposed feedback and the response with a state feedback control are presented on fig. 3. Some results obtained from a network model inducing a smaller time-delay are also presented (in dotted lines on fig. 3). These results show the efficiency of the proposed control law to stabilize the inverted pendulum when a time-varying delay occurs on the communication channel. The results obtained with a simple proportional state feedback clearly show that the system grows unstable and that this feedback can not cope with the delay. The comparison between the system response obtained for large delays and the one obtained for small delays illustrate the fact that we set the poles of the time-shifted system but not those of the actual system (while stability is ensured, the transient response depends on the delay).

\section{CONCLUSIONS}

In this paper we have investigated the problem of remote stabilization via communication networks, which is formulated as the problem of stabilizing an open-loop unstable system with time-varying delay of known dynamics.

The proposed controller includes explicitly the delay dynamics and results in a exponentially converging closed-loop system, under weak assumptions. This controller is based on a $\delta(t)$ step ahead predictor, where $\delta(t)$ is the solution of the implicit equation $\delta-\tau(t+\delta)=0$, which is shown to be solved if the time delay is bound and is transformed into a differential equation. The control scheme is applied to the TCP network, using an existing fluid-flow model.

We have also presented some experimental results showing the capability of this controller to stabilize an inverted pendulum through a TCP network.

\section{ACKNOWLEDGEMENTS}

This study was realized within the NECS-CNRS projet. The authors would like to thank the CNRS for partially funding the project. The authors would also like to thank A. Fattouh, and O. Sename, for the discussions and insights on time-delay systems.

\section{REFERENCES}

[1] S. Niculescu, E. Verriest, L. Dugard, and J.-M. Dion, "Stability and robust stability of time-delay systems: a guide tour," in Lecture Notes on Control and Information Sciences 228: Stability and Control of Timedelay Systems, L. Dugard and E. Verriest, Eds. New York: Berling Springer, 1998.

[2] E. Fridman, U. Shaked, and L. Xie, "Robust $H_{\infty}$ filtering of linear systems with time varying delay," IEEE Trans. Automat. Contr., vol. 48 , no. 1.

[3] R. Yu, "On stability of linear systems with time-varying delay: generalized lyapunov equation," IEEE AFRICON, vol. 1, pp. 569-574, 1999.

[4] E. Verriest, "Stability of systems with state-dependant and random delays," IMA Journal of Mathematical Control and Information, vol. 19, pp. 103-114, 2002.

[5] Z. Artstein, "Linear systems with delayed control: a reduction," IEEE Trans. Automat. Contr., vol. ac-27, no.4, pp. 869-879, Aug. 1982.

[6] M. Nihtilä, "Adaptive control of a continuous-time system with timevarying input delay," Systems and Control Letters, no. 12, pp. 357-364, 1989.

[7] K. Uchida, Y. Misaki, T. Azuma, and M. Fujita, "Predictive $h^{\infty}$ control for linear systems over communication channels with time-varying delays," in Proc. of the $4^{\text {th }}$ IFAC Workshop on Time Delay Systems, Rocquencourt, France.

[8] E. Witrant, C. Canudas-de-Wit, and D. Georges, "Remote output stabilization under two channels time-varying delays," in Proc. of the $4^{\text {th }}$ IFAC Workshop on Time Delay Systems, Rocquencourt, France.

[9] V. Misra, W.-B. Gong, and D. Towsley, "Fluid-based analysis of a network of aqm routers supporting tcp flows with an application to red," in Proc. of ACM SIGCOMM'00, Stockholm, Sweden, Sept. 2000.

[10] C. Hollot and Y. Chait, "Nonlinear stability analysis for a class of tcp/aqm networks," in Proc. of the $40^{\text {th }}$ IEEE Int. Conf. on Decision and Control, Orlando, Florida, USA, Dec. 2001.

[11] R. M. Corless, G. H. Gonnet, D. E. G. Hare, and D. J. Jeffrey, "On lambert's w function," University of Waterloo, Dept. Comp. Sci., Tech. Rep. CS-93-03, 1993.

[12] O. Natale, O. Sename, and C. Canudas-de-Wit, "Inverted pendulum stabilization through the ethernet network, performance analysis," Submitted to American Control Conferences, 2004. 